\title{
LA MEDICIÓN DE LA CONCIENCIA METAPRAGMÁTICA DE LOS NIÑOS: RESOLVIENDO LA AMBIGÜEDAD EN LA COMPRENSIÓN ORAL
}

\author{
MEASUREMENT OF METAPRAGMATIC CONSCIENCE \\ IN CHILDREN: RESOLVING AMBIGUITY IN ORAL \\ COMPREHENSION
}

\section{NINA CRESPO ALLENDE}

Pontificia Universidad Católica de Valparaíso, Chile

ncrespo@ucv.cl

\section{RESUMEN}

La conciencia metapragmática es aquella que posibilita al sujeto discriminar y relacionar los estímulos lingüísticos y no lingüísticos de un enunciado (Gombert, 1992). Es fundamental para la comunicación eficiente y su desarrollo es tardío, ya que emerge en la edad escolar. Las investigaciones para pesquisarla han incluido pruebas de laboratorio con pocos sujetos, pero no hay estudios que permitan describirla con poblaciones mayores. Considerando estos antecedentes y en el marco de un proyecto que busca indagar sobre la oralidad tardía y la lecto-escritura, este artículo muestra y fundamenta el diseño de un software multimedia, denominado Conciencia Metapragmática (CMP) que se aplicó a una muestra de 103 niños y niñas de 8 años de edad promedio. Su elaboración ha tomado en cuenta tres pruebas típicas de conciencia metapragmática: el referente ambiguo, las instrucciones ambiguas (Flavell, 1993) y el diálogo observado (Gombert, 1992). El análisis estadístico de los resultados ha permitido concluir que el instrumento tiene alta confiabilidad $(0,88$ según el Alpha de Cronbach). Los resultados revelan mayor dificultad en el ítem Referente Ambiguo, seguido por Instrucciones Ambiguas y Diálogo Observado, dado que en ellos la relación contexto y mensaje lingüístico es diferente. Las respuestas epi y metapragmática se dan juntas y sólo varía su frecuencia, indicando así que el paso de unas a otras ocurre en fases recursivas, más que en etapas determinadas. Además, a los 8 años las respuestas metapragmáticas son escasas, será necesario ver si existe un aumento en estudiantes de cursos superiores de Básica y de Educación Media para describir su desarrollo ontogenético.

Palabras clave: Metalingüística, metapragmática, desarrollo oral tardío, conciencia metapragmática, CMP. 


\section{ABSTRACT}

Metapragmatic conscience enables a person to discriminate and relate linguistic and non-linguistic stimuli of an utterance (Gombert, 1992). It is essential in effective communication and its development occurs late because it emerges in school age. Research that has looked at metapragmatic conscience has included laboratory tests with a few participants, but there is no research that has employed greater populations to describe it. Given these data and within the framework provided by a project that seeks to investigate both late orality and the development of reading and writing, this paper informs about the design of some multimedia software, called Metapragmatic Conscience (MPC) that was applied to 103 boys and girls of, on average, eight years of age. Its design included three typical tests on metapragmatic conscience: Ambiguous Referent, Ambiguous Instructions (Flavell, 1993) and Observed Dialog (Gombert, 1992). Statistical analysis of the results helps conclude that the instrument has a high reliability $(0.88$ according to Cronbach's alpha). Findings indicate that Ambiguous Referent was the most difficult, followed by Ambiguous Instructions and Observed Dialog, owing to the difference in the context/ linguistic message relation. Epi- and metapragmatic responses occur together and only does their frequency vary; this means that the passage from some to the others occurs in recursive phases, rather than in determined phases. Besides, since at eight years of age metapragmatic responses are scarce, their ontogenetic development could be described by looking at an increase in them among higher elementary and secondary school students.

Keywords: Metalinguistics, metapragmatics, late oral development, metapragmatic conscience, MPC.

Recibido: 27-08-2008. Aceptado: 21-02-2009.

\section{INTRODUCCIÓN}

A lo largo de su desarrollo ontogenético cada ser humano va incrementando sus habilidades cognitivas y lingüísticas. Dicho desarrollo puede ser interpretado como un incremento cuantitativo (más elementos lingüísticos, estructuras más complejas, más capacidad de procesamiento), pero también implica un aumento en la capacidad de orquestar y dirigir estos procesos. Así, Vygotsky (1995) señala que, cuando un niño crece, más que aumentar el tamaño de su memoria, se incrementa su capacidad de autodirigirla para el cumplimiento de algún objetivo propuesto. Esta autodirección de la propia cognición y el conocimiento en el cual se sustenta han sido denominados por Flavell (1993) metacognición o cognición acerca de la cognición y han sido objeto de numerosas propuestas teóricas e investigaciones empíricas.

Aplicado este concepto al uso expresivo y comprensivo del lenguaje oral, se puede concebir que todo hablante de una lengua posee un saber acerca de cómo 
usarla y guía sus intercambios lingüísticos basados en él. Autores como Gombert (1992), Verschueren (2000) y Reyes (2004) han denominado a este saber metapragmática. Especialmente Gombert (1992), ha demostrado que es un saber tardío que se desarrolla cuando la adquisición del lenguaje inicial ya ha tenido lugar. Nippold (1998) relaciona este tipo de conocimiento con el desarrollo de la oralidad en la edad escolar e incluso Puyuelo y Rondal (2003) lo consideran indispensable para el desarrollo de la habilidad de lecto-escritura. Cabe imaginar la importancia de este hecho, desde el momento que podría permitir una posibilidad de mejorar la riqueza de la lengua oral del escolar y su dominio de la lectura y la escritura, desarrollando esta habilidad más básica. Sin embargo, la mayoría de los estudios destinados a medir metapragmática se han llevado a cabo con pocos sujetos y en condiciones de laboratorio, sin que sea posible generalizar los datos a poblaciones mayores, ni dar cuenta de cómo este desarrollo tiene lugar a lo largo de la vida del niño. Para poder realizar esto, es necesario construir un instrumento que sea aplicable a una muestra considerable de niños para, a partir de allí, comprender el fenómeno desde una perspectiva más amplia.

Considerando este marco, en el presente trabajo inicialmente se esboza una fundamentación teórica con los conceptos que sirvieron de base, se explica exhaustivamente cómo se elaboró el instrumento, se detalla la metodología de aplicación y, finalmente, se exponen el análisis de los datos y las principales conclusiones y proyecciones de este estudio.

\section{MARCO TEÓRICO}

\subsection{Conciencia metalingüística y conciencia metapragmática}

Extendiendo esta noción al conocimiento y uso del propio lenguaje tanto Karmiloff- Smith (1994) como Gombert (1992) señalan la existencia de una conciencia metalingüística (entendida como conocimiento y regulación). Concretamente, Gombert (1992) sostiene que la noción de metalingüística esbozada por él es diferente a la idea de función metalingüística planteada por Jackobson (1963). Para este último, la función metalingüística tiene que ver con aquel rol que cumple el lenguaje para referirse al lenguaje. Por ejemplo, en una expresión como "La palabra casa es un sustantivo" el lenguaje estaría cumpliendo con dicha función, ya que estaría tratando acerca de sí mismo como tema u objeto de conversación. Sin embargo, para Gombert (1992) los alcances del término tienen un sesgo más psicológico. Para el autor francés, la conciencia metalingüística tiene que ver con el control consciente que cada individuo ejerce sobre su propia lengua y -en ese sentido- podría hablarse de cognición acerca del lenguaje. Bialystok (1992) considera que dicha conciencia es equiparable a uno de los dos componentes de lo 
metalingüístico: el control del procesamiento atencional, es decir, la atención selectiva a diferentes representaciones o a diferentes aspectos de una representación a medida que los problemas lingüísticos están resolviendo.

Tanto Puyuelo y Rondal (2003), como Flores-Romero, Torrado-Pacheco y Magnolia-Mesa (2006) señalan que respecto del desarrollo de las capacidades metalingüísticas existen distintas tesis. Concretamente, Flores-Romero et al. (2006) señalan dos posturas teóricas diferentes: aquella que defiende que este desarrollo es un producto de la regulación general del sistema cognitivo y otra que propone que dichas habilidades dependen exclusivamente del desarrollo del lenguaje.

En el primer grupo cabe contar autores como Bialystok (1992) y Van Kleeck, (1995), los cuales al explicar los procesos que subyacen a las habilidades metalingüísticas han propuesto que estas habilidades requieren del dominio cognitivo general. Especialmente, Van Kleeck (1994) y Levy (1999) consideraron la teoría de Jean Piaget, relacionada con los estadios de desarrollo cognitivo, para explicar la emergencia de la capacidad metalingüística. Para estos autores, cuando los niños evidencian cierto comportamiento metalingüístico éste se relaciona con la etapa de desarrollo cognitivo en la que se encuentran.

Flores-Romero et al. (2006) hacen hincapié en dos cualidades que Piaget (1987) le atribuye al pensamiento de los niños pre-escolares: La centración e irreversibilidad. La centración explica el motivo por el cual los niños durante una situación comunicativa controlan exclusivamente la fluidez del diálogo. Así, la característica de centración del pensamiento permite conjeturar que los sujetos sólo pueden concentrarse en un aspecto durante una situación y, en el caso de la comunicación, tienen en cuenta a lo más importante que es el significado. La irreversibilidad se refiere a que los sujetos pre-escolares no pueden ir de un paso $\mathrm{A}$ a un paso $\mathrm{B}$ y volver al paso A. Estos niños estarían, según Piaget (1984), en una etapa pre-operacional $\mathrm{y}$ en cuanto al lenguaje demuestran que manejan exclusivamente la forma o el significado y no pueden realizar procesos de reversibilidad que les permitan devolverse en el discurso del hablante para analizarlo (Van Kleeck, 1994). De esta manera, Levy (1999) señala que los niños menores de seis años demuestran un desempeño metalingüístico pobre, porque su forma de pensar les impide separar la forma y el significado del lenguaje en una situación comunicativa y así considerar cada elemento por separado. El hecho de que este limitado desempeño metalingüístico empiece a enriquecerse a partir de los seis años, indicaría según Van Kleeck (1994) que los sujetos han pasado a una nueva etapa del desarrollo cognitivo.

Gombert (1992), por otra parte, afirma que el desarrollo metalingüístico depende intrínsecamente del desarrollo del lenguaje. Corroborando esta idea, Chaney (1994) lleva a cabo un estudio en el cual compara niños de diferente clase social, cuyos padres tenían conceptos diferentes respecto de la alfabetización y cuyo nivel de desarrollo lingüístico era diferente. La autora concluye que el conocimiento del lenguaje es el predictor más importante del surgimiento de la 
conciencia metalingüística.

Gombert (1992), coincidiendo con Karmiloff-Smith (1994), sugiere que el desarrollo metalingüístico se produce en 3 fases: adquisición de habilidades iniciales, fase epilingüística (tomando la noción de Culioli, 1990) y fase metalingüística. Hablar de fases y no de etapas permite considerar cierta recursividad; para estos autores un niño puede estar cursando varias fases a la vez y aplicándolas de maneras diferentes en diversos microdominios y en situaciones distintas. Las dos primeras fases ocurrirían de manera más o menos sistemática, mientras que la tercera dependería del contexto.

En la adquisición de las habilidades lingüísticas iniciales, el niño aprende pares unifuncionales donde una forma lingüística corresponde a un determinado contexto. Halliday (1975) señala una etapa inicial muy similar en su enfoque funcionalista de la adquisición del lenguaje. Sin embargo, en esta visión apoyada por Karmiloff-Smith (1994) dicho proceso no sería únicamente guiado por el uso. El niño partiría con preprogramaciones innatas de las habilidades lingüísticas de base y aprendería los pares funcionales gracias a la mediación del modelo lingüístico presente en el entorno.

Dada la diversidad intrínseca del lenguaje, la estabilidad de la primera fase es cuestionada por el aumento de la extensión y complejidad de los modelos de los adultos. A partir de allí, los pares forma-función son sustituidos por formas multifuncionales, cuyo uso el sujeto administra en una suerte de ajuste automático. Esta fase es caracterizada como epilingüística y se produce por una reorganización en la memoria a largo plazo de los conocimientos implícitos acumulados dentro de la primera fase. El término epilingüístico fue acuñado por Culioli (1990), el prefijo "epi", de origen griego, se refiere a un "trabajo sobre" el idioma, que en realidad es un preliminar indispensable para llegar "más allá del” uso puramente instrumental de la lengua. En el marco de la teoría del desarrollo metalingüístico con este rótulo se identifican aquellas actividades de control que el sujeto lleva sobre su propio lenguaje pero lo hace inconscientemente, sería una fase anterior a un uso reflexivo más deliberado y explicitable.

Puyuelo y Rondal (2003) señalan dos diferencias fundamentales entre la visión de Karmiloff-Smith (1994) y la de Gombert (1992). La autora inglesa sostiene que en la etapa epilingüística se reorganiza la información de la fase anterior de adquisición. En cambio, Gombert plantea que esta reorganización surge de la necesidad de interrelacionar los conocimientos de la primera fase de adquisición, con otros recientemente descubiertos acerca de las mismas formas lingüísticas o acerca de otros elementos lingüísticos asociados con ellas que están en curso de apropiación. Además, otra diferencia apunta a cuál es la índole de la información que está actuando en esta etapa: mientras que para Karmiloff-Smith (1994) en esta fase la reorganización es impermeable a las influencias externas; Gombert (1992) afirma que -si bien la función principal de esta fase es una articulación interna 
de los conocimientos implícitos, que permitirá al sujeto el dominio funcional no consciente del sistema-, la elaboración de las reglas que rigen el uso de las formas lingüísticas viene determinada por aquello que el niño descubre acerca de dichas reglas en los funcionamientos lingüísticos en situación.

Finalmente, la etapa metalingüística está relacionada no sólo por la capacidad de reflexionar y autorregular el propio lenguaje, sino también con la posibilidad de explicitar esta conciencia que se posee del propio código. Esta fase no ocurre de forma totalmente sistemática y homogénea, los hablantes suelen ser más autorreflexivos en algunos aspectos del lenguaje que en otros y esto parece depender de las características de cada sujeto y de los diversos niveles del lenguaje que debe considerar. Así, por ejemplo, la conciencia metafonológica es una de las primeras que se desarrolla en el niño e incluso es motivada en los planes educativos preescolares, ya que ella sería facilitadora de la adquisición de la lecto-escritura inicial (Arnáiz Sánchez, Castejón Costa, Ruiz Jiménez \& Guirao Lavela, 2002).

Ahora bien, el autor francés analiza la habilidad metalingüística considerando diversos niveles de la lengua en los que podría enfocarse: fonético, semántico, sintáctico, textual y pragmático. De ellos, el presente trabajo considerará la conciencia metapragmática. Ya se señaló que Gombert (1992) y Verschueren (2000) la visualizan involucrada en el uso expresivo y comprensivo del lenguaje oral, además los autores la definen no sólo como un saber acerca de las relaciones entre contexto y mensaje lingüístico, sino también como conciencia y autocontrol de la comprensión y producción lingüística teniendo en cuenta dichos elementos.

Partiendo de lo definido a nivel metalingüístico en general, Gombert (1992) considera que todos los niños usuarios de una lengua adquieren la conciencia metapragmática en fases distintas. La de adquisición inicial se caracteriza porque en ella el niño utiliza las primeras formas en ciertos contextos. La fase epipragmática puede ser descrita de manera similar a la epilingüística, pero sus alcances se restringen a las relaciones entre lenguaje y contexto. En otras palabras, refiere actividades de control que el sujeto lleva sobre su producción y comprensión del lenguaje oral, relacionándolo con los elementos extralingüísticos, pero dicho control sería inconsciente, por lo cual podría fallar. Constituiría una fase anterior a un uso reflexivo más deliberado y explicitable propio de lo metapragmático. Cuando da respuestas enmarcadas en esta fase, el niño no discrimina explícitamente entre lo verbal y lo no verbal y, según Gombert (1992), los ve como un todo indiferenciado. Sin embargo, esta no distinción no implica necesariamente muchos errores en el uso, porque el pequeño realiza ajustes automáticos entre ambos tipos de información (posee un dominio epipragmático); sólo comete algunos traspiés de interpretación cuando dicha relación no es clara. De esta manera, puede fallar en la comprensión al enfrentarse a enunciados cuya relación con el contexto no es totalmente evidente, como el caso de las ironías o las frases hechas metafóricas no familiares.

Finalmente, la fase metapragmática se iniciaría alrededor de los 6 años e invo- 
lucraría conciencia verbalizable de la diferencia entre lo lingüístico y lo contextual $y$, en consecuencia, un control reflexivo de esta relación en una determinada situación de comunicación. Cuando empieza a manejarse metapragmáticamente, el sujeto es capaz de explicitar las relaciones forma lingüística/contexto en la producción e interpretación de los mensajes lingüísticos y, paralelamente, se produce el desarrollo de su capacidad de comprender formas no literales como la metáfora y la ironía. Para Gombert (1992) el acceso a esta fase está muy relacionado con el aumento en la capacidad de la memoria operativa. Por este motivo, la habilidad metapragmática no se aplica en forma sistemática a todas las situaciones; un sujeto podrá evidenciar un nivel de comprensión-producción lingüística evidentemente metapragmático en ciertas situaciones y uno epipragmático en otras. Es decir, el control consciente característico de esta fase no se da sólo con la edad; un niño mayor de 6 años no da respuestas metapragmáticas todo el tiempo, sino que éstas dependerán de la complejidad tanto del mensaje lingüístico como del medio contextual en el cual éste tiene lugar y con el cual se relaciona.

Para completar esta noción de metapragmática, es necesario acudir a la visión de Verschueren (2002) respecto de cuáles serían las señales que le permitirían al usuario de una lengua ajustar lo lingüístico/ lo no lingüístico cuando se autorregula. Para el autor belga los indicadores de la conciencia metapragmática funcionan de tres maneras en el lenguaje en uso: como mecanismos de anclaje que localizan formas lingüísticas en relación al contexto (deícticos, por ejemplo, los pronombres) y como señales o marcadores de interpretaciones reflexivas de los usuarios del lenguaje respecto de las actividades que realizan (indicadores léxicos, por ejemplo, adverbios oracionales) y como claves de contextualización (por ejemplo: cambios prosódicos, gestos o sonidos).

\section{METODOLOGÍA}

\subsection{Planteamiento del problema}

No existen antecedentes de medición sistemática del constructo metapragmática, relacionado con el manejo de la ambigüedad en la comunicación oral, a través del ajuste entre lo lingüístico y lo extralingüístico. Dicha medición es importante dado el rol que juega la conciencia metapragmática en la adquisición de la lectoescritura.

\subsection{Objetivos}

Objetivo general: Medir de forma sistemática el desarrollo de la conciencia metapragmática en población infantil. 
Objetivo específico: Determinar el nivel de esta habilidad en un grupo de niños y niñas chilenos, que se encuentran en tercer año básico y ya hayan logrado la adquisición inicial de la lecto-escritura.

Objetivo específico: Probar la confiabilidad de un instrumento construido para medir conciencia metapragmática (CMP) en un grupo de niños y niñas chilenos, que se encuentran en tercer año básico y ya hayan logrado la adquisición inicial de la lecto-escritura.

\subsection{Descripción de la prueba}

Dado que la población a la que se quería medir eran niños y que se buscaba observar el surgimiento de la conciencia metapragmática en ellos, era imposible evaluar esta variable con protocolos explícitos que dieran cuenta de la conciencia metapragmática por parte de los sujetos. Además, si se consideran detenidamente los antecedentes teóricos y empíricos, queda claro que puede haber conciencia aunque no haya explicitación verbal (Puyuelo \& Rondal, 2003). Por este motivo se elaboró un instrumento que buscaba indagar acerca de lo que Verschueren (2000) denominó "mecanismos de anclaje" que localizan formas lingüísticas en relación al contexto. Para ello se elaboró un software multimedia con tres tipos de ejercicios, basados en aquéllos desarrollados en la investigación experimental precedente y en los cuales se les pide a los sujetos dar cuenta de desajustes evidentes entre contexto y mensaje lingüístico (Flavell, 1993 y Gombert, 1992).

Estos ejercicios fueron de tres tipos. El primero de ellos era el de Referente ambiguo, un ítem en donde hay una expresión referencial que no especifica bien su referente. Por ejemplo, se presentan cuatro gatos, dos de ellos con collar (uno rojo y otro amarillo) y se le da la instrucción al niño de que "elija el gato con collar". A continuación en la Figura 1 presentamos un ejemplo esquematizado de este ítem. 
Imagen 1:

VOZ EN OFF:
Imagen 2: CONEJITO PREGUNTA:

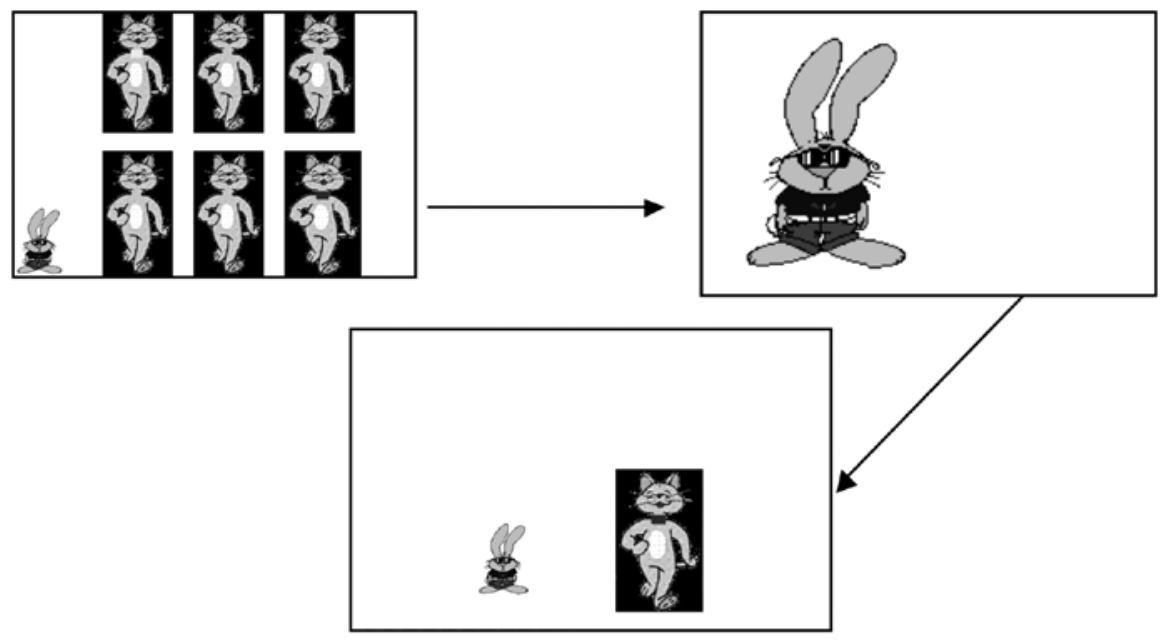

Imagen 3. CONEJITO DICE:

Está bien, hice trampa. Era el gato con collar rojo.

Figura 1. Ejemplo esquematizado del ejercicio referente ambiguo.

El niño tiene tres opciones: o elige cualquiera de los dos gatos con collar (cero punto), o manifiesta su duda pero no sabía explicarla (un punto) o explica su duda (dos puntos). En los dos últimos casos la respuesta era registrada y evaluada por el administrador de la prueba.

El segundo de ellos era el de Instrucciones ambiguas, ejercicio que consiste en darle instrucciones erróneas o incompletas al niño para participar en un juego o realizar una acción y que fue utilizado inicialmente por Flavell (1993). Como se ejemplifica en la Figura 2. 
Imagen 1:

VOZ EN OFF:

Imagen 2:

CONEJITO PREGUNTA:

Ahora descansemos. Juega conmigo a las cartas, debemos sacar una carta cada uno hasta que termine el mazo. La carta que tiene la flor señala el ganador. Gana el que tiene más cartas. ¿Entendiste? Entonces empecemos a jugar... ¿No entendiste? Entonces haz un clic aquí.

¿Me puedes explicar tu duda? (RESPUESTA ORAL

CONSIGNADA POR EL EVALUADOR)

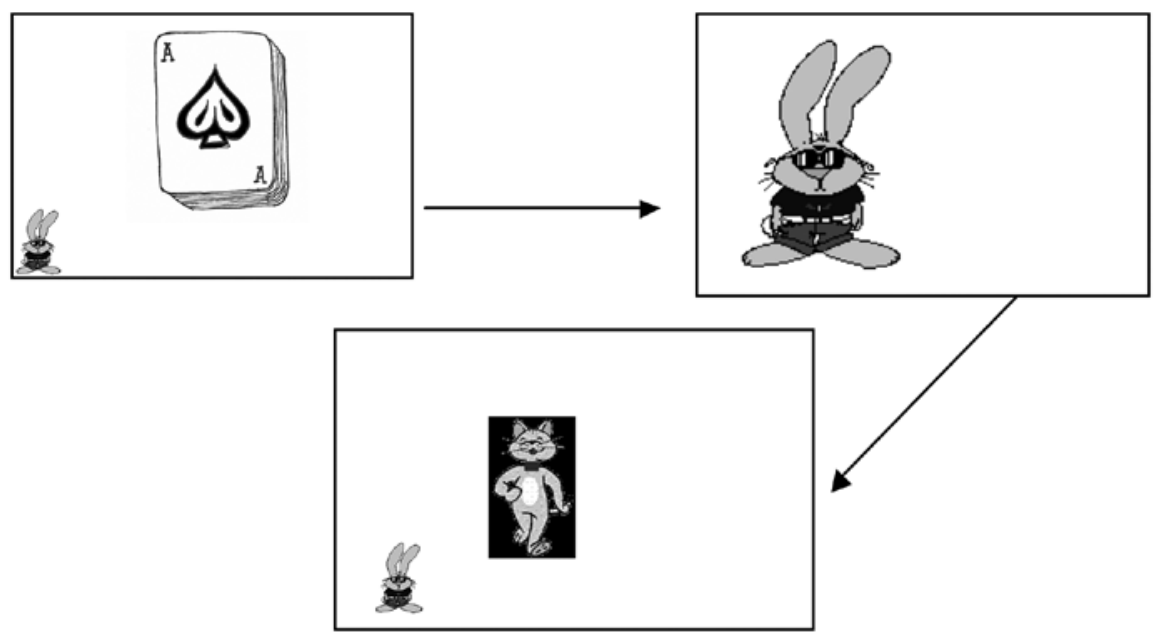

Imagen 3. CONEJITO DICE:

Está bien, no te expliqué bien. Cada uno saca una carta por vez y gana aquel que le toca la carta especial que es el As de picas. Ahora volvamos a jugar. Aprieta el botón

Figura 2: Ejemplo esquematizado del ítem instrucciones ambiguas.

Aquí, el niño también tiene tres opciones: o empieza a jugar hasta que se da cuenta que algo anda mal (cero punto), o manifiesta su duda pero no sabía explicarla (un punto) o explica su duda (dos puntos). En los dos últimos casos la respuesta era registrada y evaluada por el administrador de la prueba. 
Finalmente en los ejercicios de Diálogo Observado, el sujeto observa un mal entendido entre dos personas debido a la falta de precisión del emisor del enunciado. A continuación en la Figura 3 presentamos un ejemplo esquematizado de este ítem.
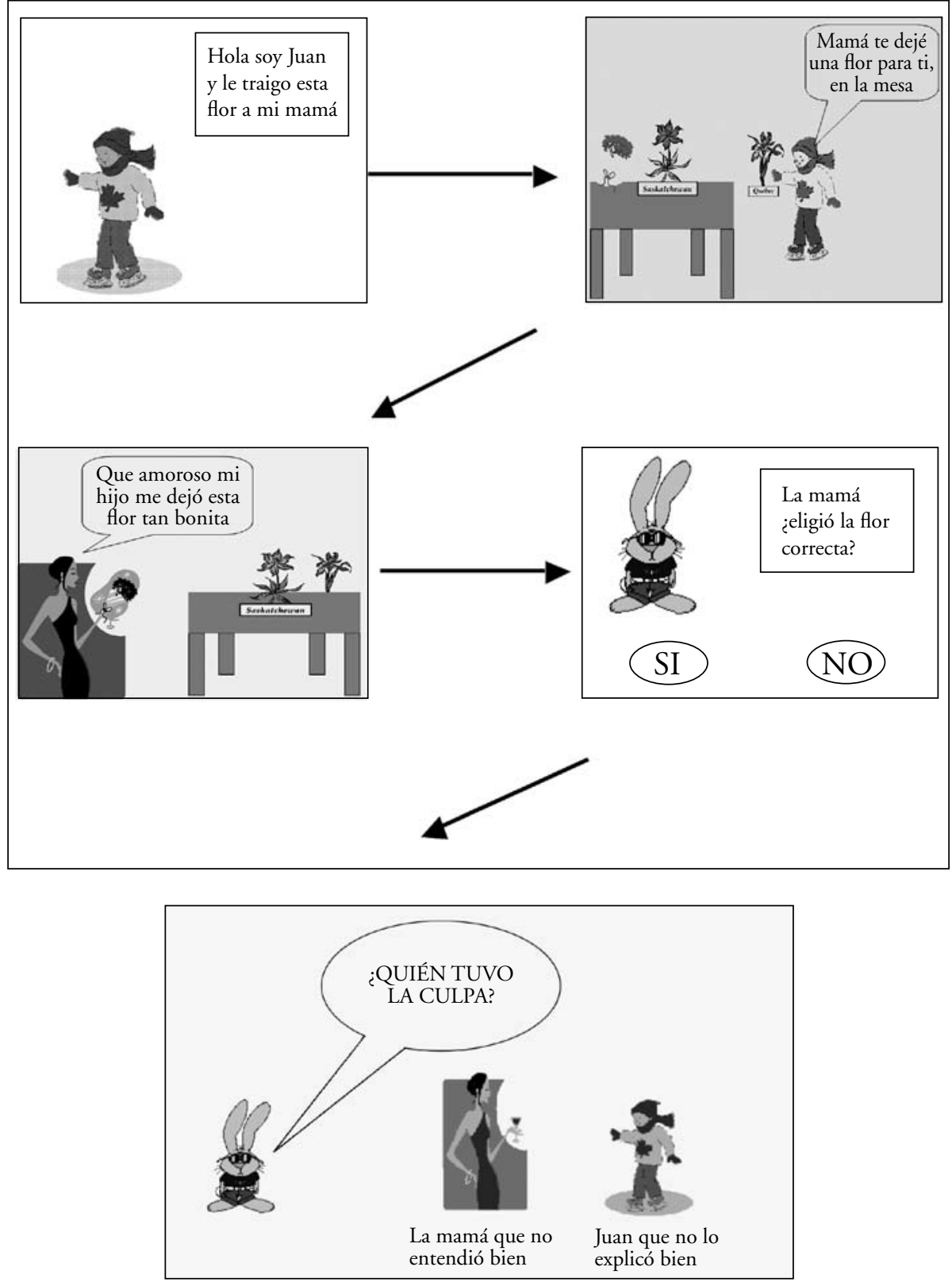

Figura 3: Ejemplo esquematizado del ítem diálogo observado. 
Si el niño señalaba que la madre había elegido la flor de Juan se le daba cero punto. Si señalaba que no, pero atribuía a la mamá el error, se le daba un punto, ya que no se daba cuenta del enunciado ambiguo de Juan. Finalmente, si podía señalar que la madre se había equivocado y que el enunciado de Juan era ambiguo, se le daba dos puntos.

En la Tabla 1 se presenta la distribución de instancias de respuesta por tipo de ejercicio y los puntajes máximos.

Tabla 1: Número de ítemes de respuesta por tipo de ejercicio y puntaje máximo.

\begin{tabular}{|l|c|c|}
\hline Pregunta & No Preguntas & Puntaje Máximo \\
\hline Referente Ambiguo & 10 & 20 \\
\hline Instrucciones Ambiguas & 9 & 18 \\
\hline Diálogo Observado & 10 & 20 \\
\hline Puntaje total & 29 & 58 \\
\hline
\end{tabular}

Como ya se señaló, el instrumento fue elaborado como un software multimedia que el niño podía manejar con el asesoramiento del evaluador. Cabe señalar que la selección de un diseño de este tipo obedeció, por un lado, a las características de los sujetos estudiados, ya que con este tipo de instrumentos, en los cuales se emula una situación de oralidad, se minimiza el esfuerzo atencional y representacional que se le exigiría a los sujetos si se utilizaran láminas e información lingüística oral. En este último caso, la tarea sería más dificultosa y no reflejaría la comprensión que ellos normalmente manifiestan en su vida cotidiana. La idea es observar su capacidad metapragmática y no de evaluar cómo responden a las dificultades planteadas por el instrumento.

Por otro lado, se eligió un software con dichas características porque esta parcial interactividad permite una mayor libertad al usuario para determinar la información que desea seleccionar. La última ventaja de emplear este tipo de instrumento frente a uno de respuesta abierta, es que el creador del software es quien decide qué opciones de participación le dará al usuario. De esta manera, será más fácil unificar y medir las respuestas que él dé (Bedoya, 1997).

\subsection{Sujetos}

El número total de sujetos en la muestra piloto fue de 103, divididos por región (V Región: Valparaíso y Viña. IV Región: La Serena y Coquimbo) y género (Femenino y Masculino), como se presenta en la Tabla 2. 
Tabla 2: Sujetos por sexo y región.

\begin{tabular}{|c|cc|c|}
\hline \multirow{2}{*}{ Sexo } & \multicolumn{2}{|c|}{ Región } & \multirow{2}{*}{ Total } \\
\cline { 2 - 3 } & IV & V & \\
\hline F & 21 & 20 & $\mathbf{4 1}$ \\
M & 30 & 32 & $\mathbf{6 2}$ \\
\hline Total & $\mathbf{5 1}$ & $\mathbf{5 2}$ & $\mathbf{1 0 3}$ \\
\hline
\end{tabular}

El instrumento CMP fue detallado en el apartado anterior, sólo resta señalar que se aplicó individualmente a cada niño y que el tiempo promedio de respuesta fue de 20 minutos.

\section{ANÁLISIS DE RESULTADOS}

\subsection{Síntesis puntaje total prueba Conciencia Metapragmática}

El análisis de los datos fue llevado a cabo por Montenegro y Pérez (2007) y en la Tabla 3 se presentan los promedios obtenidos por los sujetos en cada ítem y el promedio final. Así como el puntaje mínimo y máximo obtenido de cada tipo de ejercicio y de la prueba total, que sirven como referencia.

Tabla 3. Síntesis de puntajes promedio, máximo y mínimo obtenidos en la Prueba Conciencia Metapragmática.

\begin{tabular}{|l|c|c|c|c|}
\hline & $\begin{array}{l}\text { Referente } \\
\text { Ambiguo }\end{array}$ & $\begin{array}{l}\text { Instrucciones } \\
\text { Ambiguas }\end{array}$ & $\begin{array}{l}\text { Diálogo } \\
\text { Observado }\end{array}$ & $\begin{array}{l}\text { Instrumento } \\
\text { Total }\end{array}$ \\
\hline Promedio & 4,3 & 7,8 & 11,7 & 23,8 \\
\hline $\begin{array}{l}\text { Puntaje Máximo } \\
\text { obtenido }\end{array}$ & 20 & 17 & 20 & 55 \\
\hline $\begin{array}{l}\text { Puntaje Mínimo } \\
\text { obtenido }\end{array}$ & 0 & 0 & 2 & 8 \\
\hline
\end{tabular}

Considerando los puntajes totales por tipo de pregunta, se aprecia en la Tabla 3 que las preguntas de tipo Referente Ambiguo tienen un puntaje medio de 4 puntos, con el $50 \%$ de los alumnos con puntaje igual o inferior a 2 puntos, y arroja la más alta variabilidad con respecto al puntaje promedio, lo que indicaría una mayor dificultad con respecto a los otros dos tipo de preguntas entre los alumnos encuestados.

Los resultados obtenidos revelan una mayor dificultad en las preguntas de tipo Referente Ambiguo, seguido por las Instrucciones Ambiguas, para finalizar con las de menor dificultad, dadas por las preguntas de tipo Diálogo Observado, como se aprecia en la Figura 4. 


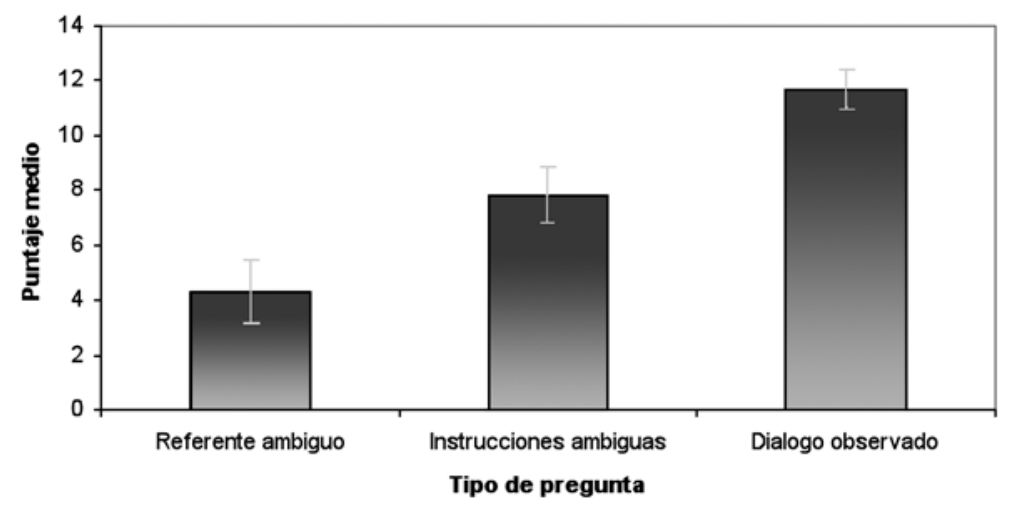

Figura 4. Puntajes promedio por tipo de pregunta, con sus intervalos de confianza del $95 \%$ de la prueba Conciencia Metapragmática.

Desde el punto de vista de la distribución de los puntajes obtenidos, Montenegro y Pérez (2007) establecieron que el 50\% de los encuestados tuvo puntaje igual o inferior a 22 puntos, es decir 2 puntos menos que el puntaje medio. De esta manera establecen que la muestra piloto tomada presenta una asimetría positiva en torno al valor medio estimado, lo que significa que hay más valores por debajo del puntaje medio, en otras palabras, el instrumento CMP resultó difícil para la población de niños de 8 años y 8 años 11 meses. Esto puede apreciarse en la Figura 5.

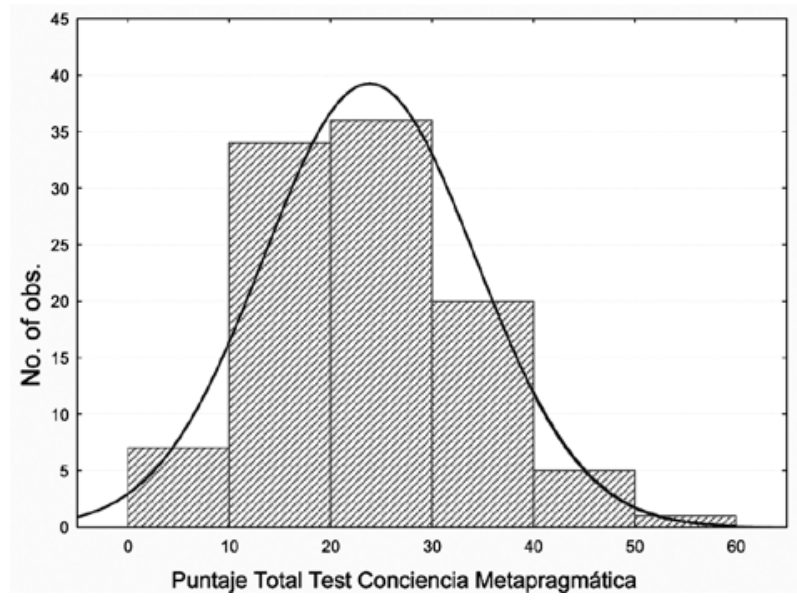

Figura 5. Histograma del puntaje de la prueba Conciencia Metapragmática. A modo de referencia se presenta la curva de una distribución normal. 


\subsection{Capacidad discriminatoria de la CMP}

Para conocer el nivel de consistencia interna del instrumento, Montenegro y Pérez (2007) estimaron el coeficiente de correlación puntual-biserial entre cada ítem y la prueba total. Tal como se muestra en la Figura 6:

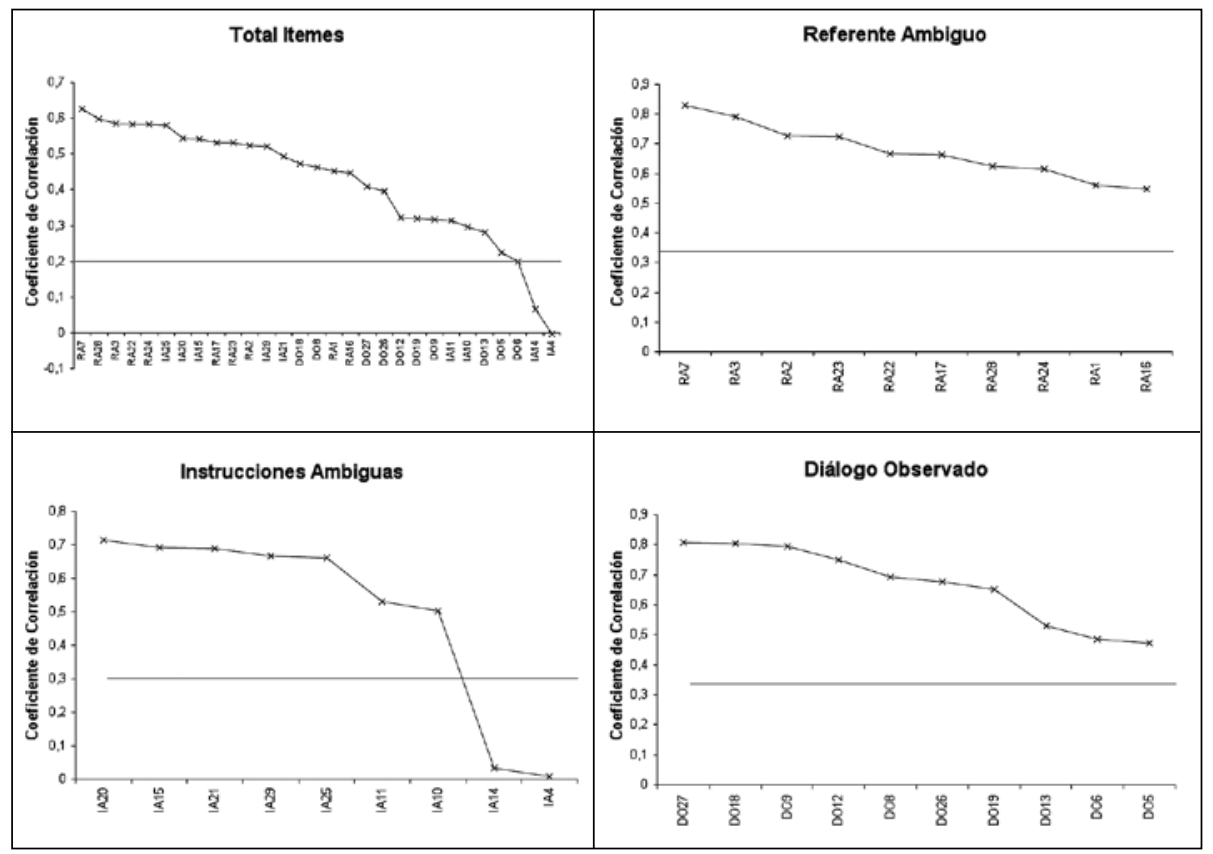

Figura 6. Coeficientes de correlación puntual-biserial entre cada ítem de la Prueba Conciencia Metapragmática y la prueba completa.

Los autores señalan que -como se observa en la Figura 6- sólo en el tipo de pregunta Instrucciones Ambiguas es necesario eliminar ítemes (4 y 14), ya que no aportan significativamente a la prueba de Conciencia Metapragmática ( Montenegro \& Pérez, 2007).

\subsection{Confiabilidad del CMP}

Finalmente, Montenegro y Pérez (2007) estimaron los índices de confiabilidad para la prueba Conciencia Metapragmática en 2 etapas: (1) con todos los ítemes y (2) habiendo eliminado los ítemes 4 y 14 ya que presentaron un coeficiente de correlación menor a $0,2(\mathrm{r}<0,2)$. 
Tabla 4: Índice de confiabilidad según el Alpha de Cronbach.

\begin{tabular}{|l|l|l|}
\hline Tipo de pregunta & $\begin{array}{l}\text { Alpha de Cronbach } \\
\text { Todos los ítemes }\end{array}$ & $\begin{array}{l}\text { Alpha de Cronbach ítemes } \\
\text { con correlación superior } \\
\text { a r } 0,2\end{array}$ \\
\hline Referente ambiguo & 0,91 & 0,91 \\
\hline Instrucción ambigua & 0,81 & 0,88 \\
\hline Diálogo observado & 0,91 & 0,91 \\
\hline Total & 0,88 & 0,89 \\
\hline
\end{tabular}

De acuerdo a Montenegro y Pérez (2007) la prueba total presenta un Coeficiente del Alpha de Cronbach (1951) superior a 0,80 que implica una muy alta confiabilidad del instrumento. Dicho índice se mantiene tanto considerando todas las preguntas como habiendo eliminado los dos ítemes con baja correlación. Dado que todas las pruebas son indicadores imperfectos de cualidades o habilidades que tratan de medir y que en todas las situaciones de prueba existen errores, el hecho de que la CMP sea muy altamente confiable implica que tiene un error estándar de medición reducido (García-Garro, Ramos-Ortega, León-Ponce \& Olivera-Chávez, 2007). Esto permitirá que sea aplicada de manera más certera en otros estudios con poblaciones similares.

\subsection{Frecuencia en el tipo de respuestas}

Como se señaló anteriormente, la CMP medía conciencia metapragmática atendiendo a dos de las tres fases recursivas en las que un sujeto puede estar transitando al mismo tiempo: a) respuestas de 0 punto, el sujeto manifiesta un control epipragmático que falla, b) respuesta de 1 punto, el sujeto es capaz de efectuar el contraste mensaje-contexto de manera epipragmática, c) respuesta de 2 puntos, el sujeto es capaz de dar cuenta de la discrepancia entre el mensaje-contexto y a la vez la reconoce explícitamente, de esta manera revela estar en la fase metapragmática. En un análisis realizado por Montenegro y Pérez (2007) se estableció que en esta muestra de niños los resultados se repartían como se señala en la Figura 7. 


\section{Total preguntas}

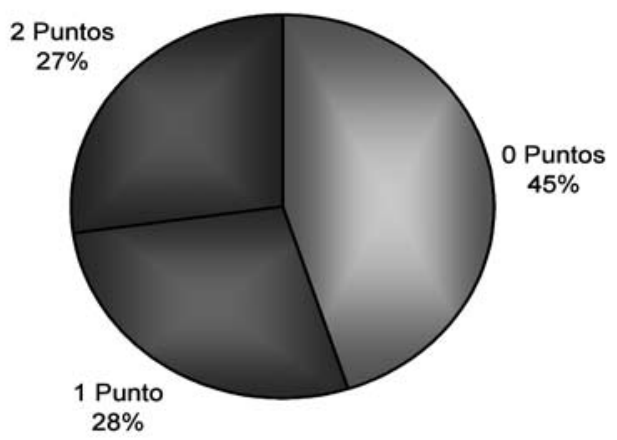

Figura 7: Porcentaje de puntajes de respuestas.

Recordando que éstas son fases no etapas, se puede decir que para la mayoría de las respuestas los niños todavía evidencian un nivel epipragmático de comprensión; ya que prevalecen las respuestas de cero punto. No obstante, dado que cada tipo de ejercicio o ítem obligaba al sujeto a establecer relaciones distintas entre contexto y enunciado y que el grupo en general resolvió mejor unos que otros, consideramos más productivo observar qué ocurría con estas respuestas al interior de cada ítem (Figura 8).

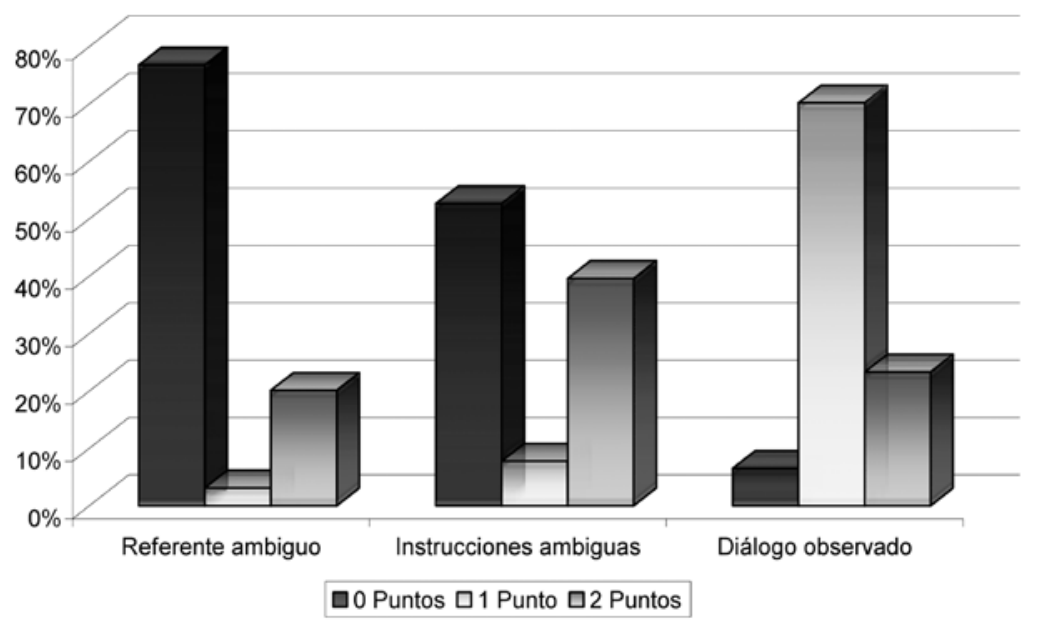

Figura 8: Porcentaje de repuestas por tipo de ejercicio. 
Se señaló anteriormente que el ítem más fácil había sido el de diálogo observado, en el cual el niño apoya sus respuestas tomando en consideración la conducta de terceros. Sin embargo, al ver la distribución de puntajes ésta puede considerarse una situación relativa. Las respuestas más frecuentes en el ejercicio Diálogo Observado son aquellas de un punto, en las cuales el niño demuestra identificar la falla en la comunicación que queda evidenciada en un error conductual, pero el reconocimiento de cuál sería el origen de la ambigüedad es mucho menor. En este sentido, los dos primeros ejercicios (Referente Ambiguo e Instrucciones Ambiguas), en los cuales las respuestas van acompañadas por un hacer del mismo sujeto, parecen producir en el individuo posiciones más extremas, acumulándose los puntajes en 0 y en 2. De esta manera, pareciera que cuando el sujeto está involucrado en el ejercicio más como protagonista que como testigo de la situación, le es más fácil detectar la no concordancia forma lingüística/contexto en la cual descansa su confusión.

\section{CONCLUSIONES}

Las conclusiones de este trabajo refieren a dos aspectos distintos de la conciencia metapragmática. Por un lado, el del instrumento construido, la CMP, y sus características como herramienta para medir la conciencia reflexiva que rige la comprensión del lenguaje en escolares, considerando no sólo lo explicitable, sino también la conciencia epipragmática que influencia en el hacer aunque no sea posible expresarla verbalmente. Por el otro, el de la habilidad epi y metapragmática en sí y en cómo ésta se manifiesta en niños que se encuentran finalizando la adquisición de la lecto-escritura inicial.

La CMP es un instrumento altamente confiable para medir conciencia metapragmática en niños de ocho años de acuerdo a los resultados de las pruebas estadísticas, a partir de allí, se puede considerar que se cuenta con un instrumento de medición óptimo para llevar a cabo otras investigaciones del tema. El hecho de que no todos los ejercicios hayan revelado el mismo nivel de dificultad, es evidencia que las relaciones entre el contexto y el mensaje lingüístico no siempre se dan de la misma manera y por ello es posible que para el niño sea más fácil dar cuenta de algunos más que de otros. En este punto parecen corroborarse las impresiones de Gombert (1992), en el sentido que la habilidad metapragmática depende de la forma en que se relacionen las informaciones de distintas fuentes y la posición que el comprendedor ocupa respecto a ellas o, como lo señala Verschueren (2002), las diferencias en su grado de saliencia.

¿Qué podemos decir de los niños de 8 años que han respondido a esta prueba? Como se señaló en el análisis de resultados, la CMP fue un tanto difícil para los pequeños estudiantes. Si se considera que esta prueba pretende medir cómo los niños son capaces de dar cuenta de un desajuste entre mensaje lingüístico y con- 
texto y, a partir de allí, demostrar que se encuentran en una fase metapragmática; es posible señalar que a los 8 años esto ocurre de manera muy parcial. Los sujetos todavía apoyan muchas de sus respuestas en un ajuste automático e incluso en aquellos casos en los cuales son observadores y -por ende- se encuentran en mejores condiciones de ver dicho desajuste, ellos logran señalar el problema pero no encuentran su origen. En otras palabras, un niño de 8 años todavía da más respuestas automáticas que conscientemente controladas. Si aceptamos lo señalado por Puyuelo \& Rondal (2003), en el sentido de que la conciencia metapragmática se encuentra relacionada con el desarrollo de la escritura, será interesente observar qué ocurre con ella en estudiantes que están en cursos superiores de Básica y Educación Media, instancias en las cuales los sujetos dominarían la lengua escrita de manera más acabada.

En este sentido, puede considerarse que la presente investigación es sólo el paso inicial, quedan varias tareas por hacer. Por un lado, ya se señaló que es indispensable observar qué pasa con la conciencia metapragmática de niños mayores y menores de ocho años. De encontrarse diferencias significativas, será posible establecer baremos de lo esperable para cada grupo de edad. Por otra parte, queda pendiente la tarea de comparar las diferencias entre la medición hecha con instrumentos de lápiz y papel y la llevada a cabo a través de este instrumento multimedia en el sentido de si facilita o no la precisión de la evaluación. Finalmente, queda precisar cuál es el rol que posee esta capacidad en el desarrollo de la oralidad tardía, sobre todo, la comprensión de significados no literales y en la lecto-escritura que se desarrolla en la situación escolar. Comprender de manera más acabada esto, nos permitiría no sólo ampliar nuestro conocimiento acerca de lo que ocurre con el lenguaje en la edad escolar, sino que también permitiría proyecciones interesantes para la didáctica de la lecto-escritura y disciplinas relacionadas con la rehabilitación de la lengua oral en niños.

\section{REFERENCIAS}

Arnáiz Sánchez, P.; Castejón Costa, J.L.; Ruiz Jiménez, M.S. \& Guirao Lavela, J.M. 2002. "Desarrollo de un programa de habilidades fonológicas y su implicación en el acceso inicial a la lecto-escritura en alumnos de segundo ciclo de Educación Infantil”, en Educación, Desarrollo y Diversidad 5, 1, pp. 29-51.

Bedoya, A. 1997. “¿Qué es la interactividad?".[On line]. Consulta: 25/02/2003. Disponible en: http://www.sinpapel.com/art001.shtml.

Bialystok, E. 1992. "Attentional control in children's metalinguistic performance and measures of field. Independence", en Developmental Psychology 28, 4, pp. 654-664.

Chaney, C. 1994. "Language development metalinguistic awarness and emergent 
literacy skills of 3 years old children in relation to social class", en Applied Psycholinguistics 15, pp. 371-394.

Culioli, A. 1990. Pour une linguistique de l'énonciation. Opérations et représentations. Tome 1. Paris: Ophrys.

Cronbach, L. J. 1951. "Coefficient alpha and the internal structure of tests", en Psychometrika 16, 3, pp. 297-334.

Flavell, J. 1993. El desarrollo cognitivo. Madrid: Aprendizaje Visor.

Flores-Romero, R., Torrado-Pacheco, M. C. E \& Magnolia-Mesa, C. 2006. "Emergencia de las capacidades metalingüísticas", en Rev. Latinoamericana de Psicología 38, 3, pp. 457-475.

García-Garro, A.J.; Ramos-Ortega, G.; León-Ponce, M.A. \& Olivera-Chávez, A. 2007. "Instrumentos de evaluación", en Revista Mexicana de Anestesia 30, 3, pp. 158-164.

Gombert, J. 1992. Metalinguistic development. Chicago: University of Chicago Press. Halliday, M. A. K. 1975. Learning how to mean: Explorations in the development of language. London: Edward Arnold (Publisher).

Jackobson, R. 1963. Essais de linguistique générale. Paris: Minuit.

Karmiloff-Smith, A. 1994. Más allá de la modularidad. Madrid: Alianza.

Levy, Y. 1999. "Early metalinguistic competence: speech monitoring and repair behavior", en Developmental Psychology 35, 3, pp. 822-834.

Montenegro, C. \& Pérez, L. 2007. Análisis Estadístico II: Test Conciencia Metapragmática. Informe interno no publicado. Proyecto FONDECYT 1070333.

Nippold, M. 1998. Later language development. Austin: Pro.Ed.

Piaget, J. 1984. Psicología del niño. Madrid: Editorial Morata.

Piaget, J. 1987. La formación del símbolo en el niño: Imitación, juego, imagen y representación. México: Fondo de Cultura Económica.

Puyuelo, M. \& Rondal, J.A. 2003. Manual de desarrollo y alteraciones del lenguaje: aspectos evolutivos y patologías en el niño y en el adulto. Madrid: Masson.

Reyes, G. 2004. "Pragmática y metapragmática: la ironía lingüística". Actas del XIV Congreso de la Asociación Internacional de Hispanistas: New York, 16-21 de julio de 2001. Isaías Lerner, Roberto Nival y Alejandro Alonso (coord.) Vol. 1, pp. 147-158.

Van Kleeck, A. 1994. Metalinguistic development. Language learning disabilities in schools-age children and adolescents; Some principles and applications. United States of America: Allyn and Bancon, pp. 53-98.

Van Kleeck, A. 1995. "Emphasizing form and meaning separately in rereading and early reading instruction", en Topics in Language Disorders 16, pp. 27-49.

Verschueren, J. 2000. "Notes on the role of metapragmatic awareness". Language use, Pragmatics 10, 4, pp. 439-456.

Verschueren, J. 2002. Para entender la Pragmática. Madrid: Gredos.

Vigotsky, L. S. 1995. Obras escogidas. (Vols. 2) Madrid: Visor. 